\title{
Salutogenesis Theory and Methods: Developments, Innovations and Next Steps
}

\author{
Lenneke Vaandrager
}

Part VIII of this book presents ideas for the future development of salutogenesis theory, research methodology, practice, teaching and capacity building. This overview chapter highlights several theoretical issues in further developing the sense of coherence (SOC) construct, followed by how we can use qualitative approaches to study the SOC, including a wide range of examples. Another important future field discussed in Part VIII is how to strengthen the salutogenic capacity of health professionals. The rapid growth of communication digitalization is also taken up in a chapter on the digital lifeworld and salutogenesis. Part VIII concludes with a chapter written by all editors of the Handbook, wrapping it up under the title Salutogenesis for Thriving Societies, which I suggest is a 'must read' for all.

\section{Theoretical Issues in the Further Development of the SOC Construct}

Chapter 53 by Jacek Hochwälder discusses theoretical issues, including:

- The dimensionality of the SOC scale, questioning whether the three components of comprehensibility, manageability and meaningfulness can be measured separately or not.

- A plea for longitudinal data to study SOC as the causal variable.

- Whether or not SOC can be strengthened and thus function as an outcome variable or not.

- The concept of domain-specific SOC.

- The concept of a boundary in the measurement of SOC.

- The dichotomization and trichotomization of SOC to investigate more thoroughly if a weak SOC or a strong SOC is crucial for health and well-being.

L. Vaandrager $(\square)$

Department of Social Sciences, Health and Society, Wageningen

University and Research, Wageningen, The Netherlands

e-mail: lenneke.vaandrager@wur.nl
- The importance of relating SOC to salutogenic outcome measures such as perceived wellness or a healthy lifestyle.

- Additional issues such as the collective SOC, measuring other central constructs in the salutogenic model and the importance of literature reviews and meta-studies.

In this chapter, excellent recommendations are given for ways forward.

\section{Qualitative Approaches to the Study of the SOC}

Antonovsky's short- and long-form SOC questionnaires have been validated in numerous languages. Therefore, there are many studies available worldwide that have used these quantitative measures. Additionally, in recent years, there has been rapid growth in qualitative studies of the SOC, as taken up in Chap. 54 by Avishai Antonovsky, Gillie Pragai Olswang, and myself. This chapter aims to address the following questions: how is the SOC measured qualitatively, when is such measurement relevant or preferable and what should we strive to achieve as we continue developing qualitative approaches? Based on our search of the literature, we report on four types of research:

- Studies that intentionally and directly measured the SOC using qualitative methodologies.

- Studies designed within the salutogenic framework that were open to analysing people's life stories or artwork and which looked for expressions which reflect the SOC.

- Studies interpreting their findings in hindsight in terms of the SOC.

- Studies that did not originate with salutogenesis in mind, and came to appreciate that something akin to the SOC had been measured. 
In the diverse array of qualitative studies, many were carried out in Scandinavian countries. Most of the qualitative researchers employed traditional research methodologies, while others applied more pioneering methods. Qualitative salutogenic research is expanding. Its theoretical and empirical contributions include demonstrating the utility in SOC research of thick descriptions of microanalytic behaviours and methods to document SOC development. An important step for the future is to develop quality criteria for qualitative salutogenic studies.

\section{Promoting Salutogenic Capacity in Health Professionals}

Eva Langeland and her colleagues have many years of experience building health professionals' salutogenic competence, as summarized in Chap. 55. From a salutogenic perspective, relational and reflective competencies are key to the success of competence building. Reflecting on and exploring one's (life) experience in a continuous learning process can enhance salutogenic competence. This learning process is also discussed in Chap. 7, where we review many years of experience teaching health promotion in postgraduate summer schools.

Chapter 55 is nicely illustrated with teaching and coaching examples drawn from: (a) a master's programme for students in various health professions, (b) salutogenic talk-therapy groups, (c) students in health promotion training programmes and (d) on-the-job training of healthcare professionals working in childcare services.

The chapter discusses the concept of 'self-tuning', referring to habitual self-sensitivity, reflection and mobilizing of resources, which can play a central role in all types of training. This chapter and Chap. 7 emphasize that trainers should strive to 'live the talk', developing their personal salutogenic capacity - in other words, do what you teach and be what you teach.

\section{The Digital Lifeworld and Salutogenesis}

In Chap. 56, Luis Saboga Nunes and colleagues explore salutogenesis in the context of the 'digital world', concerning both high- and low-resource countries. The digital world is rapidly developing and can transcend physical and financial barriers of health care and health promotion. The digital world also has many challenges, especially for equity. On the one hand, digitalization carries the risk of excluding many people - also healthcare workers - because they cannot access the digital world or do not have the technical skills to understand it (make sense of it). On the other hand, the digital world offers both new generalized resistance resources (GRRs) and specific resistance resources (SRRs) to improve population health and promote healthy lifestyles and health literacy. The authors nicely illustrate how the SOC helps people find a balance in the digital world's stressrich environment. Important steps forward in this field include work to strengthen the evidence base and to document the preconditions for a digital world that supports decision-making in health care, health behaviour change (e.g. quitting smoking) and - above all - supports empowerment and social justice.

\section{Salutogenesis for Thriving Societies}

Chapter 57 is the Handbook's 'Grand Finale', addressing the potential of salutogenesis to nurture thriving societies. The Handbook's editors reflect on the advancement of salutogenesis concerning theory development, applying the salutogenic model in community settings and helping society tackle crises such as COVID-19:

- Concerning theory development, we have come far, as illustrated in many chapters of the book, and suggestions for further theory development abound in every part of the book.

- Concerning practice, we recognize that we have not yet sufficiently realized our ambition to trigger a paradigm adjustment in the health professions, in the direction of balance between pathogenic and salutogenic orientations. Yet, there is progress in recent years, as almost a dozen chapters in the Handbook attest.

- In building salutogenesis as a transdisciplinary academic arena, an essential task for the near future is nurturing the growth and spread of the Society for Theory and Research in Salutogenesis.

Finally, in Chap. 57, we ask, is salutogenesis relevant for tackling the COVID-19 crisis? Our answer is YES because salutogenesis positively focuses on what might be seen only as a big problem. It helps us to identify individual and collective resources and imagine sustainable futures and thriving societies. 
Open Access This chapter is licensed under the terms of the Creative Commons Attribution 4.0 International License (http://creativecommons. org/licenses/by/4.0/), which permits use, sharing, adaptation, distribution and reproduction in any medium or format, as long as you give appropriate credit to the original author(s) and the source, provide a link to the Creative Commons license and indicate if changes were made.

The images or other third party material in this chapter are included in the chapter's Creative Commons license, unless indicated otherwise in a credit line to the material. If material is not included in the chapter's Creative Commons license and your intended use is not permitted by statutory regulation or exceeds the permitted use, you will need to obtain permission directly from the copyright holder.

(c) (1) 